\title{
Developmental Readiness in the Understanding of Own and Other's False Beliefs
}

\author{
Anna Amadó, Elisabet Serrat, and Francesc Sidera \\ Universitat de Girona, Plaça Sant Domènec, No. 9, 17071 Girona, Spain \\ Correspondence should be addressed to Anna Amadó; anna.amado@udg.edu
}

Received 10 July 2014; Revised 15 September 2014; Accepted 15 September 2014; Published 14 October 2014

Academic Editor: Nobuo Masataka

Copyright (C) 2014 Anna Amadó et al. This is an open access article distributed under the Creative Commons Attribution License, which permits unrestricted use, distribution, and reproduction in any medium, provided the original work is properly cited.

One of the most important milestones in the development of theory of mind is the understanding of false beliefs. This study compares children's understanding of representational change and others' false beliefs and evaluates the effectiveness of an appearance-reality training for improving children's false belief understanding. A total of 78 children ranging in age from 41 to 47 months were trained in three sessions and evaluated in a pretest and in a posttest. The results show that for children it is easier to understand representational change than false beliefs in others, and that the improvement after training was greater when starting from a higher score in the pretest. The implications of this for training in false belief understanding are discussed.

\section{Introduction}

Recognition of the mental world is possible thanks to the human capacity to understand our own mental states and those of others, which is possible because we have a theory of mind. The theory of mind (henceforth ToM) involves seeing oneself and others in terms of mental states, such as desires, emotions, beliefs, intentions, and other inner experiences that result in and are manifested in human action [1]. ToM includes a set of metacognitive competences, such as the facial recognition of certain emotions or the understanding of metaphorical expressions, irony, and lies.

For more than two decades, the most common test used to evaluate ToM has been the explicit false belief task. The origin of this task was the test of false belief (FB) understanding (Maxi Task), designed by Wimmer and Perner [2]. The results of the vast majority of the studies that assessed children's understanding of FB have suggested that, around the age of four, children are capable of understanding FB [1]. However, it is important to make a distinction between the implicit and explicit knowledge of FB. In a study conducted by Clements and Perner [3], children younger than three, despite giving incorrect verbal responses in an explicit FB task, showed an implicit understanding of FB. The results of this and other studies $[4,5]$ suggest the possibility that young children have an implicit awareness of $\mathrm{FB}$ which precedes and contributes to the explicit comprehension. It seems reasonable to consider that interference might occur between the implicit and the explicit knowledge of FB in children younger than 3 . In this sense Low [6] suggested that, for the construction of the ToM, it is necessary to understand how explicit knowledge is based on implicit knowledge and how factors such as linguistic ability make the former more accessible. According to Low [6], and following the ideas of Karmiloff-Smith, one possibility is that through the endogenous cyclical process of representational redescription, implicit representations are recoded in explicit ones, thereby allowing access to declarative verbal answers [7]. Low also suggested the possibility that, together with an implicit awareness of FB, some sets of skills, such as individual linguistic ability and cognitive flexibility, could help in the emergence of explicit understanding of FB along the implicitexplicit continuum.

This work focuses on the study of the explicit understanding of FB in children between three and four years old, and the possibility that this understanding can be improved through appearance-reality training.

Some authors assert that the acquisition of ToM implies the understanding of multiple factors, which are gradually 
acquired through a set of developmental milestones. These authors share the perspective that understands ToM as an emergent property [8] which results from the combination of a large number of features [9-11]. There are many studies of children's understanding of the different mental states involved in the ToM; however, less effort has been devoted to establishing how children gradually develop this understanding. Most such studies have specifically addressed the understanding of desires and beliefs and provided inconclusive results; while some studies have found that the understanding of desires precedes the understanding of beliefs [12-14], others do not show such a clear progression $[15,16]$. Another group of studies has focused on describing the development of ToM beyond desires and beliefs. This is the case of the work developed by Wellman and Liu [1]. The results of this work suggest that, for typically developing children, some ToM acquisitions are developed in a predictable sequence in which children progress in a series of conceptual acquisitions. Following the same line of thinking, Ketelaars et al. [17] highlighted the fact that understanding of mental representations differs according to their level of complexity, in such a way that easier understandings (e.g., emotion attribution) remain unchanged, whereas more complex measures (e.g., the understanding of mixed emotions) improve with age. This means that younger children are usually able to perform better in simpler tasks than in more advanced ones, that mental representations become more complex with age and, probably, that basic skills are a prerequisite for the successful completion of more complex tasks [18].

The studies reviewed above suggest that ToM constitutes a set of skills which appear in a gradual sequence. Based on this idea, several authors have questioned the possibility of encouraging the emergence of the abilities involved in the development of ToM, specifically in relation to FB. Some studies have focused on aspects related to ToM [19-22], while another important group of authors has focused on how language facilitates the development of FB understanding $[23,24]$. In this line, some longitudinal studies suggest that language development precedes the development of ToM, and, in this way, facilitates its progression [25-27].

In particular, the study of Hale and Tager-Flusberg [23] demonstrated that children trained in a sentential complements condition showed a significant improvement in a battery of ToM tasks. The authors concluded that mastery of this type of syntactic structure facilitates the development of ToM in preschool children, and that language has an influence on the progression of ToM. The study conducted by Lohmann and Tomasello [24] showed that children trained in both a discourse condition and a sentential complements condition improved in the understanding of FB. Moreover, a third condition where they used a combination of perspective shifting and sentential complement sentences was the best training format. In brief, the experimental studies which have used a training methodology suggest that it is possible to train children in order to foster their FB understanding [28], even in children with language disorders [29].

Another focus of special interest related to FB tasks, and more specifically unexpected content tasks, comes from the two types of questions usually asked in this test. The unexpected content task consists of showing a box whose contents have been changed before presentation (e.g., an egg box with coins inside). The box is shown closed, and the children are asked about its contents. After the children respond (they typically say that there were eggs inside), they are shown the real content (coins). The experimenter then closes the box and asks the children: (a) what they thought there was inside the box before opening it (representational change question) and (b) what another child (or a doll), who had never seen the box before, would say if she was asked about its contents (other's FB question).

From a developmental point of view, some studies propose that age is an important factor in correctly answering these questions. A study conducted by Gopnik and Astington [30] showed that the majority of infants of four and five years of age were capable of passing the representational change question, but not children aged three [31]. However, studies conducted in this area show divergent results with regard to the cognitive demands and complexities related to each question. Some suggest that both questions are of similar difficulty $[22,32]$, others state that understanding FB in others is easier than understanding representational change [30,33], and a third group of studies suggests that it is easier for children to understand representational change than FB in others $[34,35]$. The meta-analysis conducted by Wellman et al. [36] suggested that children's correct responses to FB questions for self versus others were virtually identical. Nevertheless, subsequent studies provide further evidence against this supposed synchrony in the understanding of self and others FB. For instance, the work conducted by Bender et al. [37] found that children scored higher in reporting their own FB as compared to those of other people. On the other side, Mitchell and Neal [38] found that children's understanding of others' FB was better than children's understanding of their own beliefs. Therefore, there are inconclusive results regarding the level of difficulty of the representational change question and other's FB question in the unexpected content task. It is therefore necessary to study this question in more depth.

Furthermore, we do not know studies that have evaluated, specifically, the effects of appearance-reality training in the representational change question and in the other's FB question of the unexpected content task. We consider that this new approach could provide new data to the debate about the understanding of own and other's explicit FB and about the developmental progression of these understandings in typically developing children.

The ideas outlined in previous paragraphs can be summarized as follows: (a) ToM includes a set of skills developed gradually in a predictive sequence, (b) ToM, and more specifically FB, can be trained, and (c) there are no clear results concerning the level of difficulty of the questions asked in the unexpected content task. In line with these premises, the aims of this study are to compare the level of difficulty of the questions included in the unexpected content task (the representational change question and the other's explicit FB question) and to analyze the efficacy of an appearance-reality training based on the children's understanding of FB as shown in the pretest. If one question is indeed easier than the other, 
our prediction in relation to the second aim, based on the idea that a ToM is gradually acquired, is that children who showed some degree of FB understanding in the easier question in the pretest will show further improvement in the more difficult question in the posttest, compared to children who showed no evidence of such understanding in the easier question in the pretest.

\section{Materials and Methods}

2.1. Participants. A total of 78 children ( 41 girls and 37 boys) participated in the study. The children's ages ranged from 41 to 47 months $(M=43.27$; $\mathrm{SD}=1.778)$. Apart from age, the requirements for sample selection were as follows: (a) not having completely acquired an understanding of $\mathrm{FB}$, as measured by the unexpected content task, and (b) having a language development within the norm for their age group according to the Peabody Picture Vocabulary Test [39].

2.2. Procedure and Measures. In this study we administered a pretest, training sessions, and a posttest (a sentential complement syntax test was used both in the pretest and posttest, though the results are not reported in this paper because it is outside the objectives of this study). Although three different ToM tasks were used in the posttest (change of location, unexpected content, and appearance-reality), only the results of the unexpected content task are reported here, because this was the only task which was administered in both pretest and posttest, which we detail next. Each participant attended a total of five sessions (one pretest session, three training sessions, and another posttest session), conducted individually in a quiet area of the children's school. In the first session the pretest was administered: first the unexpected content task and second the Peabody Picture Vocabulary Test. After the pretest, at intervals of three or four days, the three training sessions were conducted. Three or four days after the training was completed, the posttest was administered. In the posttest, we administered a new version of the unexpected content task. Each training session lasted from 5 to 10 minutes, while the duration of the pretest and posttest sessions ranged from 15 to 20 minutes. The parents were fully informed about the study and their consent was obtained.

Understanding of False Belief. To study children's understanding of $\mathrm{FB}$, they were given a version of the unexpected content task [30] and were asked first about their own FB concerning the contents of the box (representational change question: "What did you think there was inside the box when you first saw it?") and second, concerning the beliefs of a classmate who had not seen the contents of the box (FB in others question: "If we show this box all closed up to a friend of yours, what will he/she think there is in the box?"). These two questions were worth one point each. In the posttest, the same task was used but instead of an egg box, a Smarties box with little rocks inside was used.

Vocabulary Test. To determine whether participants were within the normal range of language development, the
Spanish version of the Peabody Picture Vocabulary Test was administered [39].

Appearance-Reality Training. This consisted of three sessions in which children were randomly shown three deceptive objects and one nondeceptive object (see [28]). The deceptive objects (e.g., a marker pen in the shape of a tube of pomade) had a certain appearance (a tube of pomade), but when looked at more closely turned out to be something else (a marker pen). The experimenter showed and labelled first the deceptive part of the object (e.g., "Look, name of the girl/boy, a tube of pomade!"), and then its real part (e.g., "Now look, a marker pen!"). This part of the training was done three times. After that, a puppet of the Little Red Riding Hood appeared and the experimenter showed the object to the puppet (first the deceptive and then the real part). In this second part it was the puppet who labelled the appearance and the reality of the deceptive objects. First, the experimenter showed the deceptive part of the object and he said: "Look puppet!", and the Little Red Riding Hood answered: "A tube of pomade!" Then, the experimenter showed to the puppet the real part of the object and said: "Now look!" and the puppet said: "Oh! A marker pen!" In this part the labelling was not repeated. In the two parts (interaction with the child and interaction with the puppet) the appearance and the reality of the objects were labelled without using mental state verbs or sentential complement structures.

\section{Results}

Of the 78 participants, 52 obtained a score of zero in the pretest unexpected content task, while 26 had a score of one. Of these 26 children, 25 had responded correctly to the own FB question, and only one responded correctly to the other's FB question. The Wilcoxon test shows that this difference is significant $(Z=4.707, P=.000)$.

In comparing the results of the children who scored one in the pretest $(N=25)$ with those who obtained zero $(N=52)$, we did not take into account the child who scored one in the other's FB question, in order to make the group who scored one more homogeneous. Before making any other comparison, we used the Mann-Whitney $U$ test to compare the age and level of receptive vocabulary of the children who scored zero in the pretest with that of the children who scored one (see Table 1). We found no differences between the two groups, either for age $(Z=-.227, P=.821)$ or for the results in the Peabody test $(Z=-1.572, P=.116)$.

3.1. Representational Change Question in the Unexpected Content Task. Approximately $71 \%$ of the children who obtained a score of zero in the pretest responded correctly to the representational change question in the posttest (see Table 2). In the group of children who obtained a score of one in the pretest, the percentage was $96 \%$. The Chi-square test shows that this difference was significant $\left(\chi_{2}^{2}=6.331, P=\right.$ .012). So the children who obtained a score of one in the pretest obtained better results in the representational change question in the posttest than did the children who obtained 
TABLE 1: Age and raw scores in the Peabody Picture Vocabulary Test (PPVT) as a function of the children's scores in the pretest.

\begin{tabular}{lcc}
\hline & Age $^{1}$ & PPVT $^{2}$ \\
\hline $\begin{array}{l}\text { Pretest score }=0 \\
N=52\end{array}$ & $43.27(1.8)$ & $27.06(5.8)$ \\
$\begin{array}{l}\text { Pretest score }=1 \\
N=25\end{array}$ & $43.12(1.6)$ & $28.67(9.9)$ \\
\hline Notes: ${ }^{1}$ age in months and ${ }^{2}$ direct marks in the PPVT. &
\end{tabular}

TABLE 2: Number of children who gave correct answers in each question in the posttest unexpected content task as a function of the children's scores in the pretest.

\begin{tabular}{lcc}
\hline & $\begin{array}{c}\text { Representational } \\
\text { change }^{1}\end{array}$ & Other's false belief \\
\hline $\begin{array}{l}\text { Pretest score }=0 \\
N=52\end{array}$ & $37(71.1 \%)$ & $12(23.1 \%)$ \\
$\begin{array}{l}\text { Pretest score }=1 \\
N=25\end{array}$ & $24(96 \%)$ & $12(48 \%)$ \\
\hline
\end{tabular}

Note: ${ }^{1}$ percentages in parentheses.

a score of zero, so the initial differences were maintained between both groups.

3.2. Other's False Belief Question in the Unexpected Content Task. In the group who obtained a score of zero in the pretest, only $23.1 \%$ of the children $(M=.23$; $\mathrm{SD}=.42)$ responded correctly to the other's FB question in the posttest (see Table 2). Conversely, $48 \%$ of the participants who obtained a score of one in the pretest $(M=.48 ; \mathrm{SD}=.51)$ succeeded in the other's FB question in the posttest. The Chi-square test showed that this difference was statistically significant $\left(\chi_{2}^{2}=\right.$ $4.888, P=.027$ ). Considering that both groups started with a score of zero, this means that children who had a score of one in the representational change question of the pretest were more likely to improve their understanding of this question.

The Wilcoxon test also showed that the children's improvement in the other's FB question between the pretest and the posttest was significant for both groups of children (see Figure 1), those who scored 0 in the pretest $(Z=$ $-3.464, P=.001)$, and those who had a score of one $(Z=$ $-3.464, P=.001)$.

The results obtained in the other's FB question in the posttest did not correlate significantly with the age of the children $(r=.011, P=.922)$ or their score in the Peabody test $(r=-.006, P=.967)$. On the other hand, there was a correlation between the children's score in the pretest and their score in the other's FB question in the posttest $(r=$ $.345, P=.002)$.

3.3. Improvement in the Unexpected Content Question. Of the group of 52 participants who obtained a score of zero in the pretest, a total of 37 children improved their score in the posttest ( 25 children scored one point and 12 scored two points). Of the 25 participants who had a score of one in the pretest, a total of 12 improved in the posttest (by answering

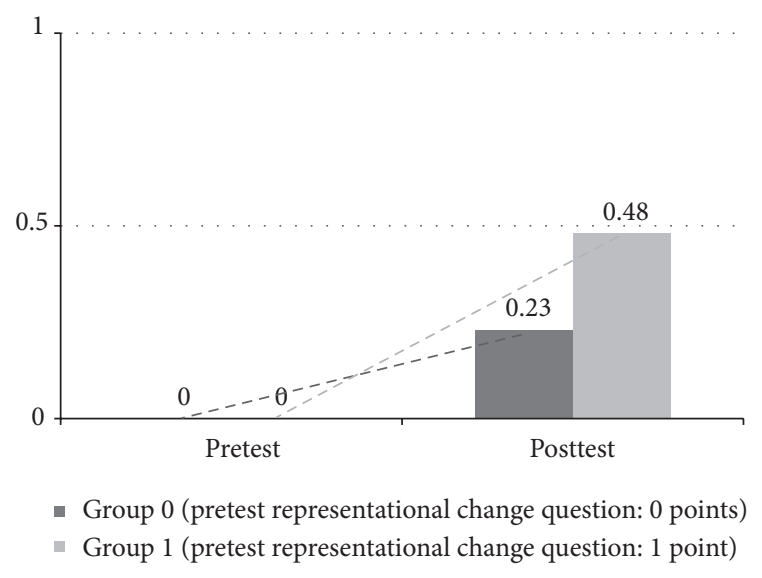

FIGURE 1: Correct responses (means) to the other's false belief question in the pretest and posttest.

correctly both questions), while 12 participants maintained their scores and one child scored zero. It is noteworthy that, apart from the participant who was excluded from the analyses, no participant correctly answered the other's FB question without having responded correctly to the representational change question.

\section{Discussion}

In this study we have focused on one of the most important developmental milestones in the development of ToM: the understanding of FB. The unexpected content task, which is one of the standard tasks designed to evaluate FB in children, includes two questions: a representational change question and other's FB question. The studies carried out to date have provided inconclusive results regarding the existence of a developmental distance between these two abilities.

The results of our study indicate that, in the pretest, there was a higher percentage of children who answered correctly the representational change question than children who answered correctly the other's FB question, which is in line with some of the previous studies $[34,35]$. Of the 26 children who obtained one point in the pretest, only one child correctly answered the question about others' FB and incorrectly answered the representational change question. On the other hand, 25 children answered the representational change question correctly and the other's FB question incorrectly.

These results suggest that the representational change question implies less difficulty than the other's FB question. This highlights the major difficulty three-year-olds have in understanding FB in others, at least in the standard task we have used in our study. To answer the representational change question correctly, the child needs to understand that the representation of reality can change while reality itself remains unchanged. Children must understand that their own beliefs change according to their knowledge of reality. However, to answer the other's FB question correctly, children must understand that perceptions of the same reality may 
differ between people. The greatest difficulty in answering correctly the other's FB question could be that it is necessary to consider the knowledge of reality of another child, whereas to answer correctly the representational change question, the child only needs to go back in time to access the memory of their own past belief, based on a previous state of knowledge. There is no need for the child to understand that their own FB was caused by a wrong expectation of reality.

Another possible explanation for the results found in our study could come from the theoretical proposition of Perner et al. [35]. These authors have suggested that, in addition to the idea we have already proposed, in order to answer the other's FB question correctly it is necessary for the child to understand that their imaginary friend will give the wrong answer while wanting to give the right one. Without the ability to understand true values in conflict it would seem to be impossible to understand how another child can give an incorrect answer despite wanting to respond correctly. On the other hand, to answer the representational change question correctly, it would not seem to be necessary to understand true values in conflict. This could explain why it is easier for children to answer correctly a question involving representational change question in themselves than to understand FB in others. However, as Mitchell and Neal [38] have suggested, the self-other difference could be due to a difference in the relevant information the children are provided with. The fact that the children in our study found the self question easier could be because they had had access to their previous FB, but not to the FB of the observer.

The nature of the task and the question format are crucial factors in the understanding of FB [40]. The results concerning the developmental distance between the understanding of the representational change and the understanding of FB in others were inevitably conditioned by the type of questions we used. In this connection, it could be interesting for future studies to test whether young children have the same difficulty in understanding a change of a belief in themselves, as measured in the representational change question, as a change of a FB in another person. To assess this latter understanding, children could observe another person opening a box with unexpected contents and then be asked about the previous belief of the person who opened the box. In this way, the question asked to the children would be the same for self and other.

As well as identifying in our study developmental distance in favour of representational change understanding, we also evaluated the efficacy of an appearance-reality training to improve FB understanding. A significant percentage of the children from our study (63.6\%) have improved significantly with the training. This result is consistent with previous studies showing that appearance-reality training is effective in improving FB in children $[23,24,28]$. According to Low [6] it is possible that this training helps children to redescribe implicit knowledge of FB in terms of explicit knowledge.

We have compared the efficacy of the training according to the degree of FB understanding in the pretest, measured by the marks gained in the representational change question in the unexpected content task. The results showed that children who obtained one point in the pretest performed better in the posttest than their peers who obtained zero points in the pretest. Then, our results show that children starting with a higher mark in the pretest performed better in the posttest and therefore benefited more from the training. It could be that the children who scored one point in the pretest were at a stage similar to the zone of proximal development proposed by Vygotsky [41], in which a relatively small amount of external support (the support offered by the training) could be sufficient to cause a substantial cognitive change. Using the concept employed by Bird [42], we can say that the children who obtained one point in the pretest showed a developmental readiness that, added to the training effects, allowed them to achieve a substantial change in their understanding of FB. This developmental readiness acts as a precursor to explicit false belief understanding and makes children with this knowledge be more susceptible to take advantage of the intervention. It is important to consider the possibility that if the assistance had been given before the existence of this state of developmental readiness in the children, they could not have taken advantage of the training to the same degree. That is what might have happened to some of the children who scored zero in the pretest.

\section{Conclusions}

To sum up, the results of our study suggest that an understanding of representational change is acquired prior to understanding $\mathrm{FB}$ in others, and that the children who answered the representational change question successfully in the pretest benefited more from the training than the children who did not show this understanding. Therefore, it seems that in FB understanding there is an optimal developmental point at which children's understanding can be improved, which is probably more related to understanding of representational change than chronological age. This finding would be significant in terms of developing training programs aimed at developing children's ToM. In addition, our study shows that FB understanding can be facilitated through appearance-reality training and that it is therefore possible to influence the normal course of children's development, in a way which follows a sequence that is to some extent predictable. For these reasons, this topic requires further investigation.

\section{Conflict of Interests}

The authors declare that there is no conflict of interests regarding the publication of this paper.

\section{Acknowledgments}

This research was supported by a grant from the University of Girona (Reference SING2010B/9) and a predoctoral grant 2009-2013 (Reference BR09/19) from the same university. The authors thank all the schools and pupils who participated in the study. 


\section{References}

[1] H. M. Wellman and D. Liu, "Scaling of theory-of-mind tasks," Child Development, vol. 75, no. 2, pp. 523-541, 2004.

[2] H. Wimmer and J. Perner, "Beliefs about beliefs: representation and constraining function of wrong beliefs in young children's understanding of deception," Cognition, vol. 13, no. 1, pp. 103$128,1983$.

[3] W. A. Clements and J. Perner, "Implicit understanding of belief," Cognitive Development, vol. 9, no. 4, pp. 377-395, 1994.

[4] K. K. Oniski and R. Baillargeon, "Do 15-month-old infants understand false beliefs?" Science, vol. 308, no. 5719, pp. 255258, 2005.

[5] V. Southgate, A. Senju, and G. Csibra, "Action anticipation through attribution of false belief by 2 -year-olds," Psychological Science, vol. 18, no. 7, pp. 587-592, 2007.

[6] J. Low, "Preschoolers' implicit and explicit false-belief understanding: relations with complex syntactical mastery," Child Development, vol. 81, no. 2, pp. 597-615, 2010.

[7] A. Karmiloff-Smith, Beyond Modularity: A Developmental Perspective on Cognitive Science, The MIT Press, Cambridge, UK, 1992.

[8] M. Tomasello, M. Carpenter, J. Call, T. Behne, and H. Moll, "Understanding and sharing intentions: the origins of cultural cognition," Behavioral and Brain Sciences, vol. 28, no. 5, pp. 675691, 2005.

[9] L. Barrett, P. Henzi, and R. Dunbar, "Primate cognition: from "what now?"to 'what if ?"' Trends in Cognitive Sciences, vol. 7, no. 11, pp. 494-497, 2003.

[10] V. Grimm and S. F. Railsback, Individual-Based Modeling and Ecology, Princeton University Press, Princeton, NJ, USA, 2005.

[11] M. S. Ison-Zintilin and G. S. Morelato-Giménez, "Habilidades socio-cognitivas en niños con conductas disruptivas y víctimas del maltrato," Universita Psychologica, vol. 7, no. 2, pp. 357-367, 2007.

[12] K. Bartsch and H. Wellman, Children Talk about the Mind, Oxford University Press, New York, NY, USA, 1995.

[13] A. Gopnik and V. Slaughter, "Young children's understanding of changes in their mental states," Child Development, vol. 62, no. 1, pp. 98-110, 1991.

[14] H. M. Wellman and J. D. Woolley, "From simple desires to ordinary beliefs: the early development of everyday psychology," Cognition, vol. 35, no. 3, pp. 245-275, 1990.

[15] P. Mitchell, Acquiring a Conception of Mind: A Review of Psychological Research and Theory, Psychology Press, Hove, UK, 1996.

[16] J. Perner, "The many faces of belief: reflections on Fodor's and the child's theory of mind," Cognition, vol. 57, no. 3, pp. 241-269, 1995.

[17] M. P. Ketelaars, M. van Weerdenburg, L. Verhoeven, J. M. Cuperus, and K. Jansonius, "Dynamics of the theory of mind construct: a developmental perspective," European Journal of Developmental Psychology, vol. 7, no. 1, pp. 85-103, 2010.

[18] E. H. M. Sterck and S. Begeer, "Theory of mind: specialized capacity or emergent property?" European Journal of Developmental Psychology, vol. 7, no. 1, pp. 1-16, 2010.

[19] J. Amsterlaw and H. M. Wellman, "Theories of mind in transition: a microgenetic study of the development of false belief understanding," Journal of Cognition and Development, vol. 7, no. 2, pp. 139-172, 2006.
[20] M. Appleton and V. Reddy, “Teaching three year-olds to pass false belief tests: a conversational approach," Social Development, vol. 5, no. 3, pp. 275-290, 1996.

[21] W. A. Clements, C. L. Rustin, and S. McCallum, "Promoting the transition from implicit to explicit understanding: a training study of false belief," Developmental Science, vol. 3, no. 1, pp. 8192, 2000.

[22] V. Slaughter and A. Gopnik, "Conceptual coherence in the child's theory of mind: training children to understand belief," Child Development, vol. 67, no. 6, pp. 2967-2988, 1996.

[23] C. M. Hale and H. Tager-Flusberg, "The influence of language on theory of mind: a training study," Developmental Science, vol. 6, no. 3, pp. 346-359, 2003.

[24] H. Lohmann and M. Tomasello, "The role of language in the development of false belief understanding: a training study," Child Development, vol. 74, no. 4, pp. 1130-1144, 2003.

[25] J. W. Astington, “The future of theory-of-mind research: understanding motivational states, the role of language, and realworld consequences," Child Development, vol. 72, no. 3, pp. 685687, 2001.

[26] J. Astington and J. Baird, Why Language Matters for Theory of Mind, Oxoford University Press, London, UK, 2005.

[27] M. E. Bermúdez-Jaimes and L. V. Sastre-Gómez, "Falsa creencia y desarrollo semántico del lenguaje en niños de 2 a 4 años," Universitas Psychologica, vol. 9, no. 3, pp. 849-861, 2010.

[28] E. Serrat, C. Rostan, E. Vallès-Majoral, M. Esteban, F. Sidera, and J. Serrano, "Labelling improves false belief understanding. A training study," The Spanish Journal of Psychology, vol. 16, pp. 1-14, 2013.

[29] E. Serrat, J. Serrano, A. Amadó et al., "Entrenar la comprensión de la falsa creencia en niños con alteraciones del lenguaje," Revista de Logopedia, Foniatría y Audiología, vol. 32, pp. 109119, 2012.

[30] A. Gopnik and J. W. Astington, “Children's understanding of representational change and its relation to the understanding of false belief and the appearance-reality distinction," Child Development, vol. 59, no. 1, pp. 26-37, 1988.

[31] P. D. Zelazo and J. J. Boseovski, "Video reminders in a representational change task: memory for cues but not beliefs or statements," Journal of Experimental Child Psychology, vol. 78, no. 2, pp. 107-129, 2001.

[32] K. Sullivan and E. Winner, "When 3-year-olds understand ignorance, false belief and representational change," British Journal of Developmental Psychology, vol. 9, no. 1, pp. 159-171, 1991.

[33] M. Naito, S. Komatsu, and T. Fuke, "Normal and autistic childrens understanding of their own and others false belief: a study from Japan," British Journal of Developmental Psychology, vol. 12, no. 3, pp. 403-416, 1994.

[34] C. Moore, K. Pure, and D. Furrow, "Children's understanding of the modal expression of speaker certainty and uncertainty and its relation to the development of a representational theory of mind," Child Development, vol. 61, no. 3, pp. 722-730, 1990.

[35] J. Perner, S. R. Leekam, and H. Wimmer, "Three-year olds' difficulty with false belief: The case for a conceptual deficit," British Journal of Developmental Psychology, vol. 61, no. 5, pp. 125-137, 1987.

[36] H. M. Wellman, D. Cross, and J. Watson, "Meta-analysis of theory-of-mind development: the truth about false belief," Child Development, vol. 72, no. 3, pp. 655-684, 2001. 
[37] P. K. Bender, F. Pons, P. L. Harris, and M. de Rosnay, "Do young children misunderstand their own emotions?" European Journal of Developmental Psychology, vol. 8, no. 3, pp. 331-348, 2011.

[38] R. W. Mitchell and M. Neal, "Children's understanding of their own and others' mental states, Part B: understanding of others precedes self-understanding for some false beliefs," British Journal of Developmental Psychology, vol. 23, no. 2, pp. 201-208, 2005.

[39] L. Dunn, E. Padilla, D. Lugo, and L. Dunn, Test de Vocabulario en Imágenes Peabody. Spanish Version of the Peabody Picture Vocabulary Test-Revised, American Guidance Service, Circle Pines, Minn, USA, 1986.

[40] L. J. Moses and J. H. Flavell, "Inferring false beliefs from actions and reactions.", Child development, vol. 61, no. 4, pp. 929-945, 1990.

[41] L. Vygotsky, Mind in Society: The Development of Higher Psychological Processes, Harvard University Press, Cambridge, UK, 1978.

[42] L. Bird, "Beyond readiness: new questions about cultural understandings and developmental appropriateness," in The Praeger Handbook of Education and Psychology, pp. 428-438, Praeger, Westport, Ireland, 2006. 

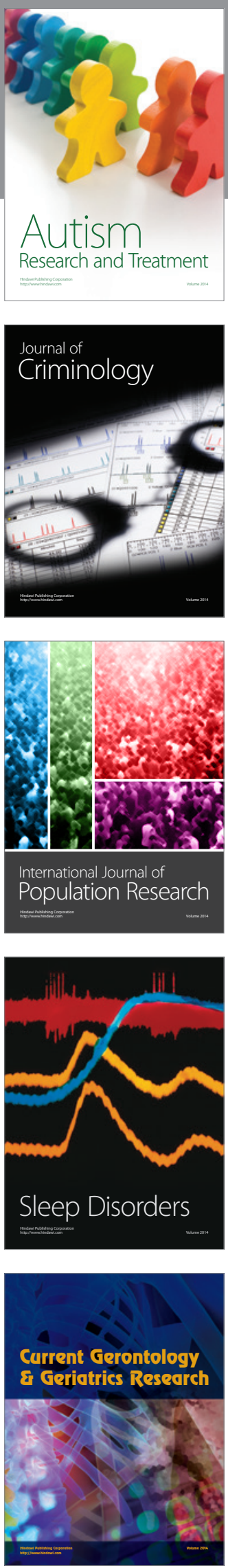
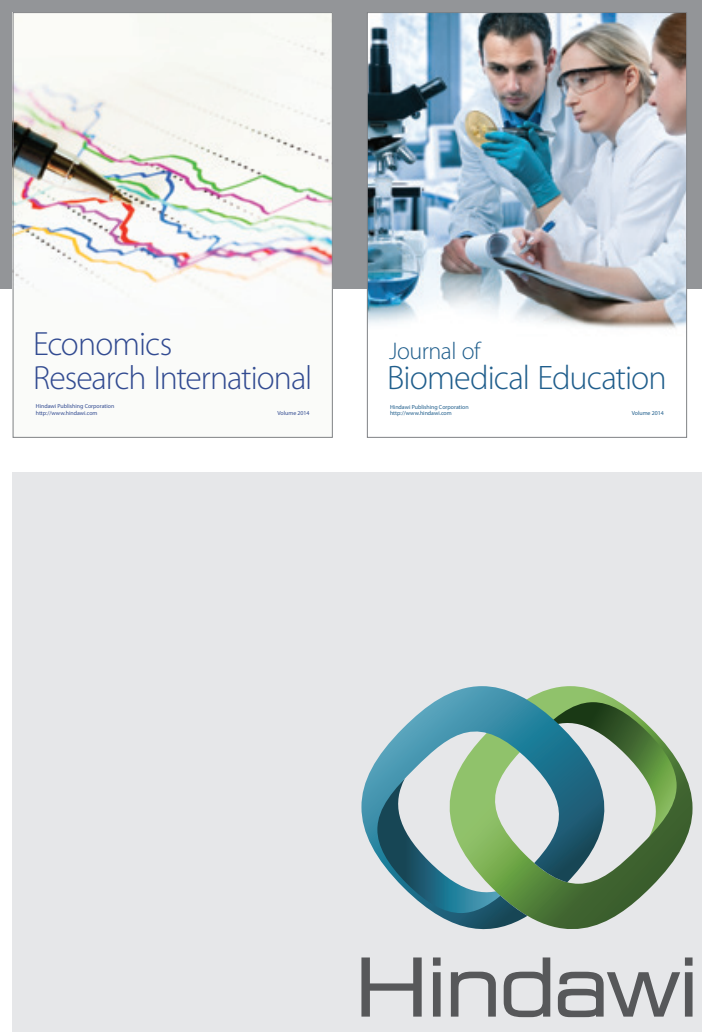

Submit your manuscripts at

http://www.hindawi.com
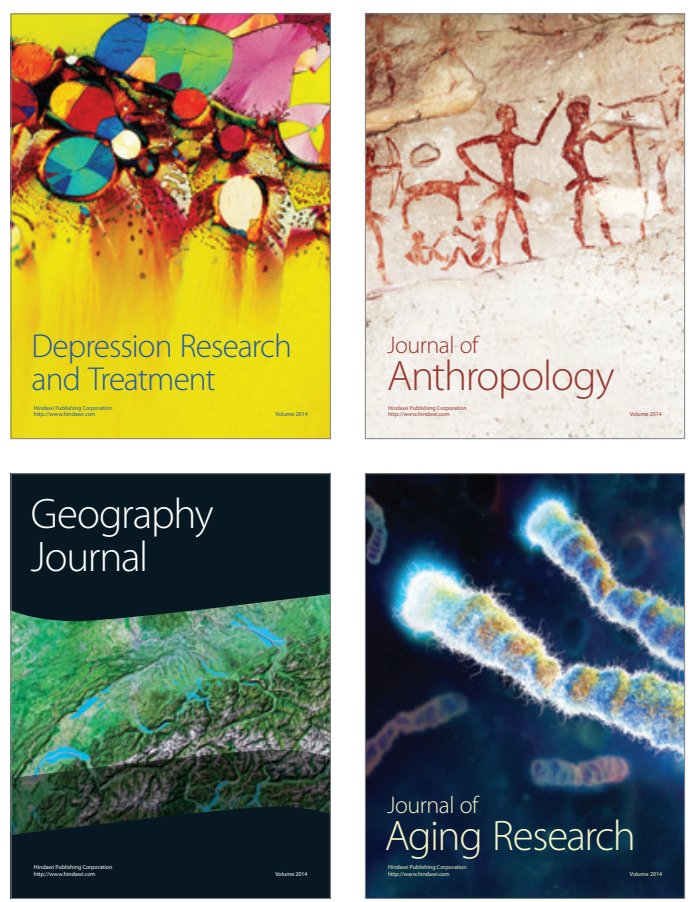
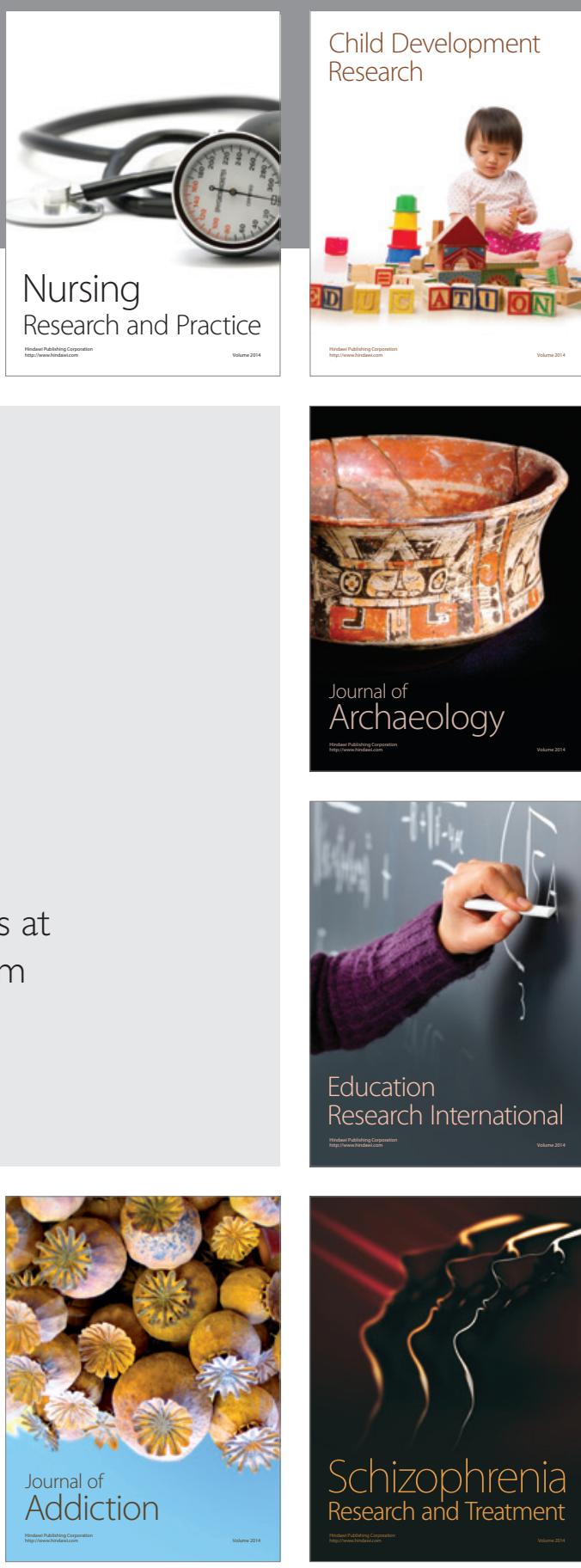

(D)
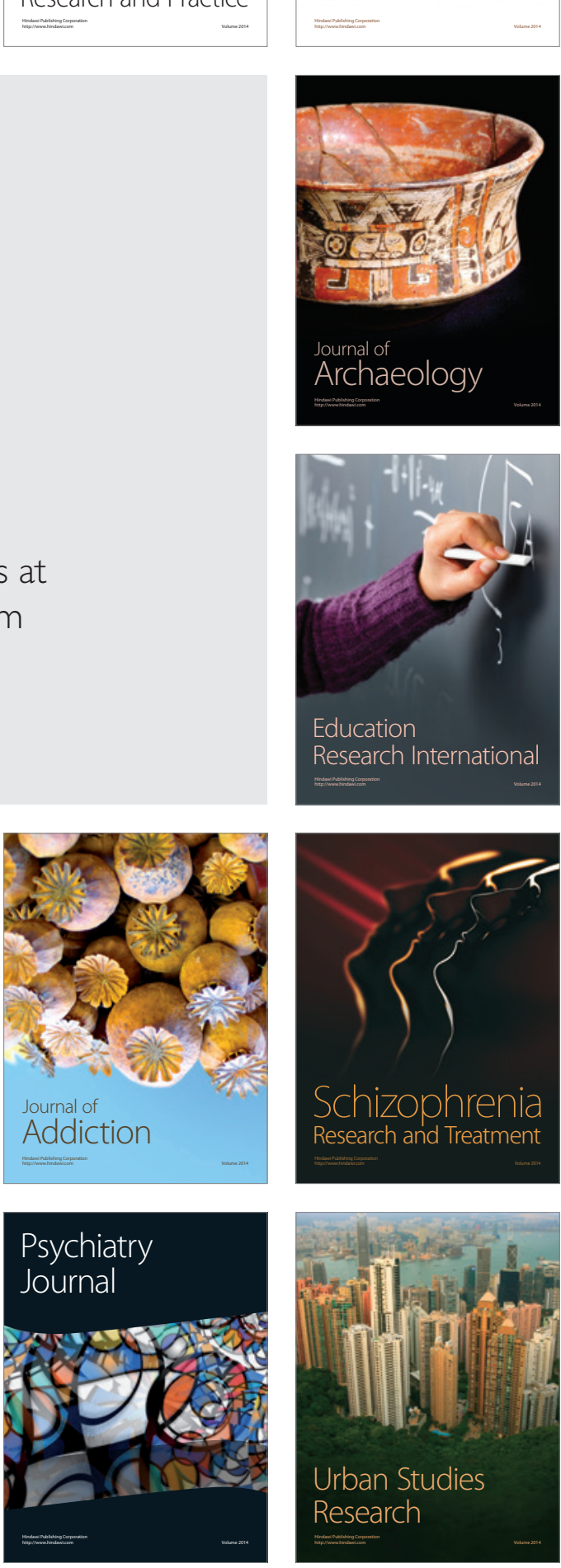